\title{
Morfometría Mandibular en Adultos a través de una Radiografía Panorámica
}

\author{
Mandibular Morphometry in Adults by Panoramic Radiographic \\ "Ramón Fuentes; *** Ruth Prieto; ${ }^{* * *}$ Héctor Silva; "Thomas Bornhardt \& *F. Cuevas
}

FUENTES, R.; PRIETO, R.; SILVA, H.; BORNHARDT, T. \& CUEVAS, F. Morfometría mandibular en adultos a través de una radiografía panorámica. Int. J. Morphol., 26(2):415-418, 2008.

RESUMEN: Los procedimientos clínicos a nivel del hueso mandibular en Odontología, en las etapas de diagnóstico, planificación y tratamiento, son rutinarios. Para poder realizar dichas fases, es relevante conocer en forma precisa la anatomía mandibular, con el fin de minimizar los riesgos en los procedimientos anestésicos, quirúrgicos y protésicos. El uso de radiografías panorámicas es cada vez más frecuente, las cuales permiten un estudio de la anatomía mandibular bilateral. El objetivo del presente estudio fue analizar la morfología de la mandíbula a través radiografías panorámicas, estudiando específicamente la ubicación de estructuras, como el foramen mandibular en relación a los diferentes márgenes de la rama de la mandíbula. El foramen mental se analizó en relación con el cuerpo mandibular, y se revisaron las longitudes de la rama y cuerpo mandibulares. Se estudiaron 50 radiografías panorámicas, sin diferencia de género, el rango de edad estaba entre 25 y 72 años y sin patología disfuncional. Los forámenes mandibular y mental, en este estudio no mostraron variaciones significativas. La ubicación del foramen mandibular se encontró estable en ambos lados.

PALABRAS CLAVE: Mandíbula; Radiografías panorámicas; Forámenes.

\section{INTRODUCCIÓN}

En la práctica odontológica son frecuentes los procedimientos clínicos a nivel del hueso mandibular, en las etapas de diagnóstico, planificación y tratamiento. Para poder realizar éstas, se requiere conocer en forma detallada la anatomía de la mandíbula para minimizar las dificultades que se producen en procedimientos anestésicos, quirúrgicos y protésicos (Perin et al., 2004). En la atención de pacientes es cada vez más frecuente el uso de radiografías panorámicas, las que permiten un estudio de la anatomía del hueso mandibular bilateral (Habets et al., 1987; Hansson et al., 1993; Okeson, 2003).

En radiografías panorámicas, se han reportado variaciones anatómicas del canal y foramen mandibular y del foramen mental (Perin et al.). Bezuur et al., 1989 estudiaron factores etiológicos de los trastornos temporomandibulares encontrando diferencias significativas en la altura vertical del proceso condilar, en pacientes con origen artrogénico y/ o miogénico de dolor orofacial.

Silva \& Fuentes (2004) en un estudio de morfometría del proceso condilar y de la rama mandibular, encontraron variaciones en la altura vertical condilar en mujeres y de la rama mandibular en hombres. Se han reportado variaciones del proceso condilar en pacientes con una oclusión cubierta (Ari-Demirkaya et al., 2004). En implantología oral, las radiografías panorámicas son de uso frecuente y permiten obtener datos importantes como cantidad y calidad del hueso (Cicero \& Daudt, 2003). Conocer en forma detallada los diferentes reparos óseos del hueso mandibular, permite evitar accidentes quirúrgicos e identificar zonas de seguridad para la colocación quirúrgica de implantes (Peñarrocha, 2001).

En los procedimientos anestésicos que se realizan a nivel mandibular es fundamental conocer la ubicación de los forámenes a través de los cuales entra y sale el nervio alveolar inferior (Perin et al.; Evers \& Haegerstam, 1983).

\section{MATERIAL Y MÉTODO}

Se estudiaron 50 radiografías panorámicas de pacientes, sin diferencia de género, el rango de edad estaba entre

* Depto. Odontología Integral, Facultad de Medicina, Universidad de la Frontera, Chile.

** Depto. Pediatría y Cirugía Infantil, Facultad de Medicina, Universidad de la Frontera, Chile.

*** Depto. Ciencias Básicas, Facultad de Medicina, Universidad de la Frontera, Chile. 
25 y 72 años, sin patología disfuncional. Las radiografías fueron tomadas en un Ortopantomógrafo Proline CC Plan Meca y fueron realizadas por el mismo operador y con una técnica estándar. El proceso de revelado de efectuó en una máquinaDurr X 24 pro. Se utilizaron placas radiográficas Kodak T-MAT G/RA.

Para realizar las mediciones se utilizó un negatoscopio y los trazos se hicieron sobre una transparencia puesta sobre la placa radiográfica. Las mediciones se realizaron con un Cáliper digital marca Mitutoyo (Fig. 1). Se realizó un trazado con el fin de medir las distintas variables de la mandíbula, como se describe a continuación (Fig. 2).
Se trazó una línea (L1) tangente al borde inferior del cuerpo de la mandíbula, paralela a la horizontal. La línea L2 se trazó tangente al margen lateral de la rama y perpendicular a L1. La línea L3 se trazó tangente al margen medial de la rama y perpendicular a L1. La línea L4 se trazó tangente a la parte más profunda de la incisura mandibular y perpendicular a L2 y paralela a L1. Se trazó una línea (L5) a través de la línea media determinada por la espina nasal anterior, y perpendicular a L1.

Los forámenes mandibular y mental fueron marcados en sus puntos más centrales. A partir de estos trazados y puntos de referencia, se realizaron las siguientes mediciones:
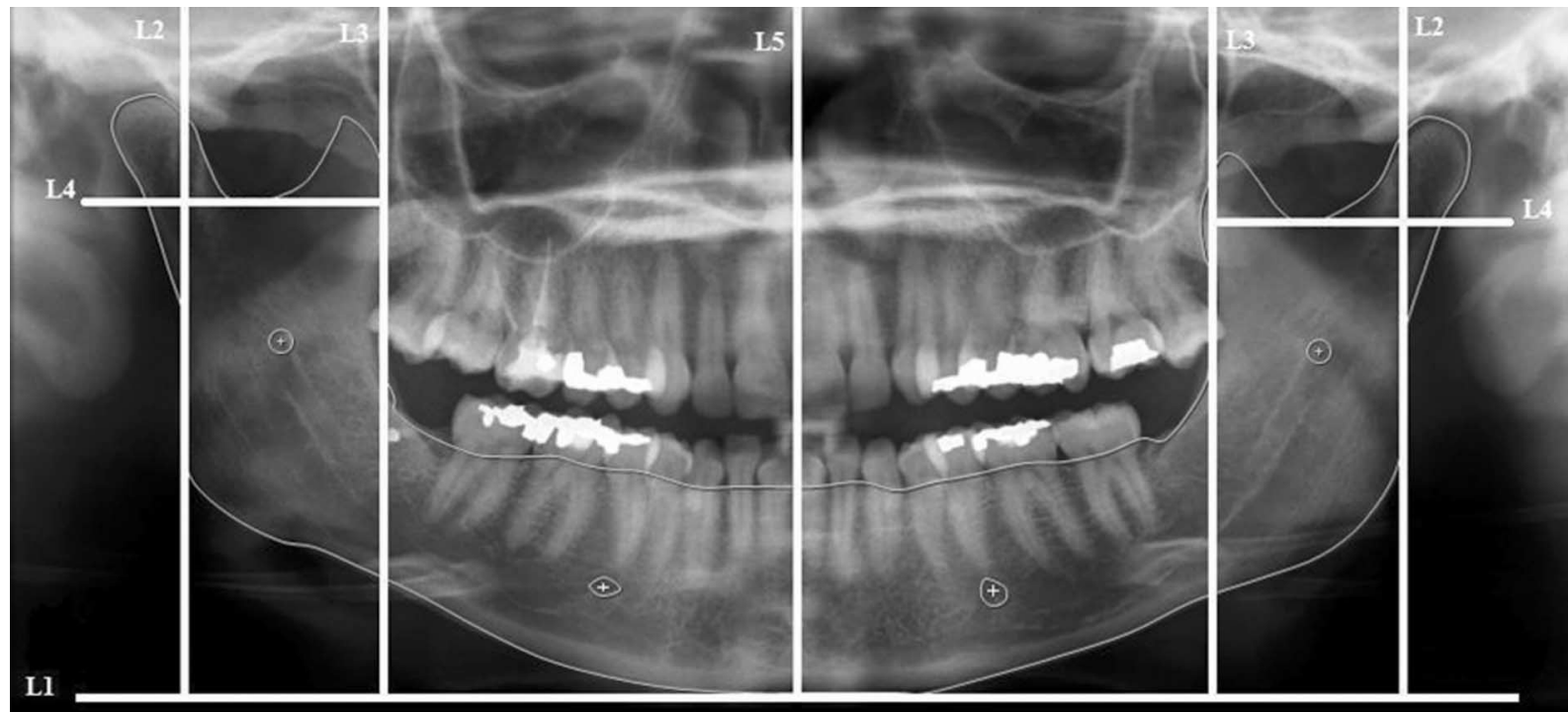

Fig.1. Radiografía panorámica de mandíbula, con los diferentes puntos de medición.

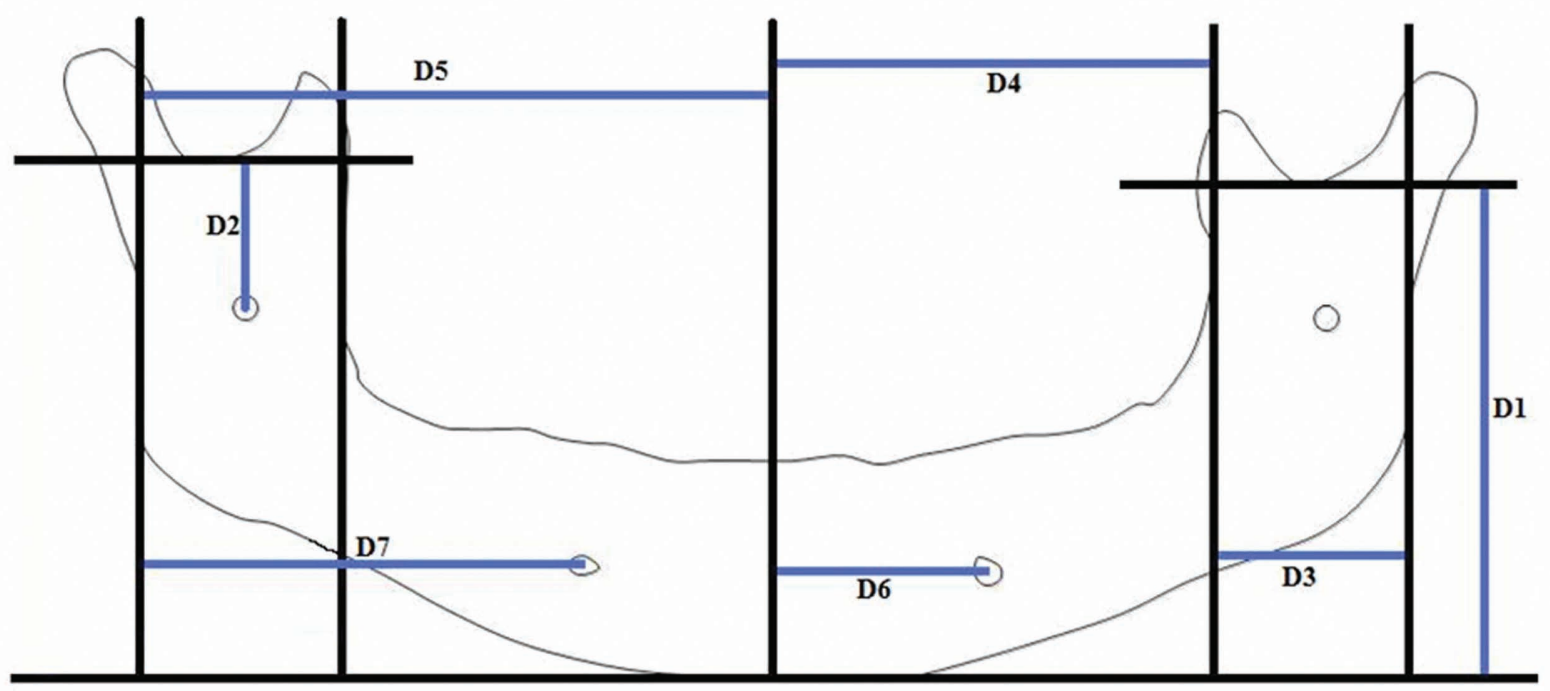

Fig. 2. Esquema de la mandíbula con el trazado de las diferentes variables medidas. 
- Altura de la rama mandibular (D1), desde L4 a L1.

- Distancia entre la incisura mandibular y el foramen mandibular (D2), desde L4 al centro del foramen mandibular.

- Ancho de la rama mandibular (D3) desde L2 a L3.

- Ancho del cuerpo mandibular (D4) desde L5 a L3.

- Ancho de cuerpo más rama mandibular (D5) desde L5 a L2.

- Distancia entre el foramen mental y línea media (D6) desde L5 al centro del foramen mental.

- Distancia entre el foramen mental y el margen posterior de la ra-ma (D7) L2 al centro del foramen mental.

Las distancias medidas fueron paralelas al eje mayor de la medición. Las mediciones se realizaron en forma bilateral con el fin de compararlas.
Los resultados de las mediciones fueron tabulados y analizados mediante pruebas estadísticas, utilizando el software STATATM 9.0.

\section{RESULTADOS}

Los resultados de las mediciones realizadas están descritos en la Tabla I.

Tabla I. Valores de centralización y significancia de las variables de una muestra de 50 radiografías panorámicas de mandíbulas de la ciudad de Temuco-Chile.

\begin{tabular}{llllllll}
\hline & \multicolumn{3}{c}{ IZQUIERDA } & \multicolumn{5}{c}{ DERECHA } \\
\cline { 2 - 8 } & $\mathbf{n}$ & Media & D.S & $\mathbf{n}$ & Media & D.S & p \\
\hline Altura de Rama & 50 & 57.0 & 6.68 & 50 & 56.1 & 6.16 & 0.4584 \\
Incisura Mandibular - Foramen Mandibular & 50 & 18.2 & 4.78 & 50 & 17.1 & 4.48 & 0.5568 \\
Ancho de Rama & 50 & 31.0 & 4.41 & 50 & 31.1 & 4.33 & 0.8570 \\
Ancho Cuerpo & 50 & 74.7 & 5.94 & 50 & 74.9 & 5.42 & 0.8645 \\
Ancho Rama mas Cuerpo & 50 & 105.7 & 8.00 & 50 & 106.1 & 7.63 & 0.8355 \\
Foramen Mentoniano - Línea Media & 50 & 31.9 & 4.81 & 50 & 32.9 & 4.27 & 0.2749 \\
Foramen Mentoniano - Borde Posterior & 49 & 73.7 & 7.50 & 50 & 73.2 & 7.45 & 0.7102 \\
\hline
\end{tabular}

\section{DISCUSIÓN}

Se pudo comprobar que las radiografías panorámicas reproducen en forma detallada las diferentes estructuras anatómicas, siendo su identificación simple (Perin et al.). Esto permite un análisis bilateral de la anatomía mandibular (Habets et al.; Hansson et al.; Okeson).

En cuanto a la ubicación de los forámenes mandibular y mental no mostraron variaciones significativas, a diferencia de lo indicado por Perin et al., quienes reportaron variaciones anatómicas. Las mediciones realizadas no aportan diferencias estadísticamente significativas para determinar eventuales factores de riesgo de trastornos temporomandibulares; son más relevantes las mediciones a nivel condilar realizadas por Bezuur et al. La altura de la rama no tuvo variaciones significativas, a diferencia de un estudio anterior de Silva \& Fuentes.

Las mediciones realizadas entregan información relevante en cuanto a referencias anatómicas de la ubicación del foramen mental, para la planificación de cirugías de implantes en el sector antero-inferior de la mandíbula, reafirmando que es el sector más seguro y permitiendo procedimientos de bajo riesgo. (Cicero \& Daudt; Peñarrocha).
En la ubicación del foramen mandibular no hubo diferencias significativas, reafirmándose que al utilizar referencias anatómicas adecuadas, los procedimientos anestésicos tronculares del nervio alveolar inferior deberían ser exitosos (Perin et al.; Evers \& Haegerstam, 1983).

FUENTES, R.; PRIETO, R.; SILVA, H.; BORNHARDT, T. \& CUEVAS, F. Mandibular morphometry in adults by panoramic radiographic. Int. J. Morphol., 26(2):415-418, 2008.

SUMMARY In dentistry, the clinical procedures at mandible level, in the diagnosis, planification and treatment stages are rutinaries. To develop these phases it's necessary to know the mandible's anatomy to minimize the risks in anesthetic, surgical and prosthetic procedures. The use of panoramic radiographies is becoming more common every day and they allow studying the bilateral mandible anatomy. This study's goal was to analyze the mandible's morphology throw panoramic radiographies. This was got by the study of the structures location such as the mandible's foramen in relation to the different margins of the mandible's branches. The mentonian foramen was analyzed in relation to mandible body and the sizes of the mandible body and the mandible branch were checked. Both genders 50 panoramic radiographies were analyzed. The age range was between 25 to 72 years old. They didn't present dysfunctional pathology. In this study, the mandible and mental foramens didn't show significant variations. The mandible foramen's location was found stable in both sides.

KEY WORDS: Mandible; Panoramic Radiographic; Foramens. 


\section{REFERENCIAS BIBLIOGRÁFICAS}

Ari-Demirkaya, A.; Biren, S.; Ózkan, H. \& Küçukkeles, J. Comparison of deep bite and open bite cases: normative data for condylar positions, paths and radiographic appearances. J. Oral. Rehabil., 31:213-24, 2004.

Bezuur, J. N.; Hansson, T. M. \& Wilkinson, T. M. The recognition of craniomandibular disorder -an evaluation of the most reliable signs and symptoms when screening for CMD. J. Oral Rehabil., 16:367-72, 1989.

Cicero, J. \& Daudt, W. Implantes Oseointegrados. Cirugía y Prótesis. Ed. Artes Médicas, Brasil, 2003.

Evers, H. \& Haegerstam, G. Manual de anestesia local en Odontología. Salvat, Barcelona, 1983.

Habets, L. L. M.; Bezzur, C. P.; Van Ooij, C. P. \& Hansson, T. L. The orthopantmogram, an aid diagnosis of temporomandibular joint problems. I. The factor of vertical magnification. J. Oral Rehabil., 14:475-80, 1987.

Hansson, T. L.; Chiristensen, C. \& Wagnon, D. Physiotherapie bei craniomandibulären Funktionsstörungen. Quintessenz Verlags-GmbH, Berlin 1993.

Okeson, J. Tratamiento de oclusión y afecciones temporomandibulares. $5^{\text {a }}$ ed. Elsevier, Madrid, 2003.

Peñarrocha, M. Implantología Oral. Ed. Ars Médica, Barcelona, 2001.

Perin, C. P.; Suzuki, A. M. M.; Fernandez, A.; Westphalen, F. H. \& Schussel, J. L. Importância das vaiaçoes antómicas dos canais mandibulares e sus implicaçóes clínicas. J. Bras. Clin. Odontol. Int., 8(44):144-6, 2004.

Silva, M. H. \& Fuentes, F. R. Morfometría del proceso condilar y rama de la mandíbula en individuos adultos de la ciudad de Temuco, Chile. Int. J. Morphol., 22(2):169-72, 2004.
Dirección para correspondencia:

Prof. Dr. Ramón Fuentes F.

Facultad de Medicina,

Universidad de La Frontera

Casilla 54-D

Temuco - CHILE

Email: rfuentes@ufro.cl

Recibido : 12-11-2007

Aceptado: 21-04-2008 